\title{
GeV to Multi-TeV Cosmic Rays: AMS-02 Status and Future Prospects
}

\author{
Martin Pohl* \\ DPNC and Center for Astroparticle Physics (CAP Genève) \\ Université de Genève \\ E-mail: martin.pohl@cern.ch
}

Due to recent observations, there is a renewed interest in $\mathrm{GeV}$ to multi- $\mathrm{TeV}$ cosmic ray measurements. Spectrum and composition measurements may in particular indicate dark matter contributions to the positron flux. Future instruments and their expected contributions to questions like cosmic ray origin, acceleration and propagation, as well as non-standard sources like dark matter or strangelets will enrich the observational base in the near future. The final flight set-up of the AMS-02 instrument is presented in detail and its performance is assessed. Its expected sensitivity to unusual sources of cosmic rays is estimated.

35th International Conference of High Energy Physics

July 22-28, 2010

Paris, France

${ }^{*}$ Speaker. 


\section{Introduction}

Recent data about antiparticle spectra have caused a renewed interest in cosmic ray properties and composition in the $\mathrm{GeV}$ to multi- $\mathrm{TeV}$ region. While the antiproton to proton ratio is fully compatible with secondary production [1], the positron to electron flux ratio [2] as well as the sum of electron and positron fluxes [3] show an unusual shape. Instead of falling monotonously, it appears that positrons account for a rising fraction of lepton spectra above a few GeV. This phenomenon seems to persist up to $100 \mathrm{GeV}$ without a visible cut off. These data cause a wide spread debate on possible astrophysical and/or dark matter sources.

In this situation, new experiments like AMS-02 and PEBS [4] promise to extend the range of high accuracy measurements to the multi-TeV range. This is particularly important since the cut off energy of the anomaly indicates the energy range of the cosmic accelerator in case of an astrophysical origin, or the mass of a hypothetical dark matter particle.

After a period of political uncertainty, the AMS-02 detector is now assured a ride to the International Space Station in 2011. In the following I will review the original configuration of the AMS-02 detector with a superconducting magnet, and present its performance as measured in a beam test at CERN in February 2010. I then retrace the arguments that led to the decision to change for a permanent magnet of lesser field strength and present the performance of the final flight configuration.

\section{Configuration and performance of AMS-02 with the superconducting magnet}

Fig. 1a) shows the layout of AMS-02 detector around and inside the superconducting magnet of $0.84 \mathrm{~T}$ central field strength. The heart of the detector is a spectrometer with six double layers of double sided silicon strip detectors inside the magnet bore, on three ultralight support planes. Two larger layers fitted into the magnet conical flanges complete the spectrometer. The spatial resolution of all layers is $10 \mu \mathrm{m}$ in the bending plane (see Fig. 1c). The spectrometer is sandwiched between two crossed double layers of scintillator planes to identify the direction of passing particles and to measure their velocity and absolute charge. Above, a high performance Transition Radiation Detector (TRD) improves the distinction between light from heavy particles with a rejection power of more than 100 at $90 \%$ electron efficiency. Below the spectrometer, a Ring Imaging Cherenkov Counter (RICH) measures the velocity of relativistic particles with a resolution of $\delta \beta / \beta \simeq 10^{-3}$ (see Fig. 1b). The set up is completed by a lead-scintillating fiber electromagnetic calorimeter which helps in electron-proton distinction by shower shape. It is also an independent detector for high energy photons. The energy resolution of the calorimeter is 2.5 to $3 \%$ as shown in Fig. 1c). In total, the particle identification devices have a combined rejection power for protons agains positrons of about 1 in $10^{6}$ at $400 \mathrm{GeV}$.

Having completed commissioning of the superconducting magnet and the extensive beam tests summarized above, the detector was transported to ESTEC to undergo electromagnetic emission and thermal vacuum tests. The electromagnetic emission fulfilled the NASA upper limit in the full frequency range between $30 \mathrm{~Hz}$ and $50 \mathrm{MHz}$. Detector performance was also nominal even at extreme temperatures down to $-90^{\circ} \mathrm{C}$, as verified with cosmic muons. The magnet helium vessel stabilized according to the thermal model and reached the stability criterion of $d T / d t<$ 
a)

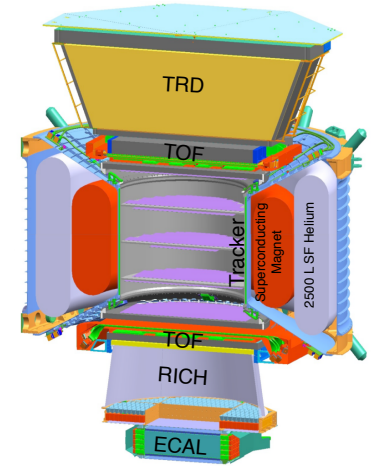

b)
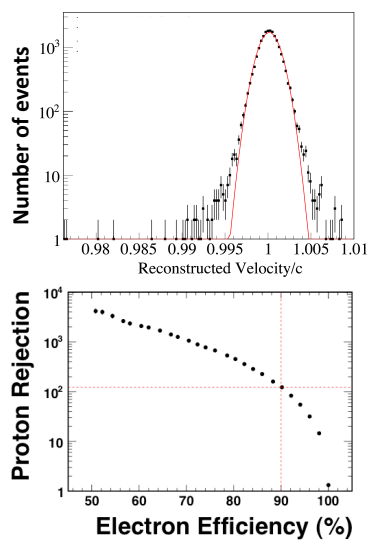

c)
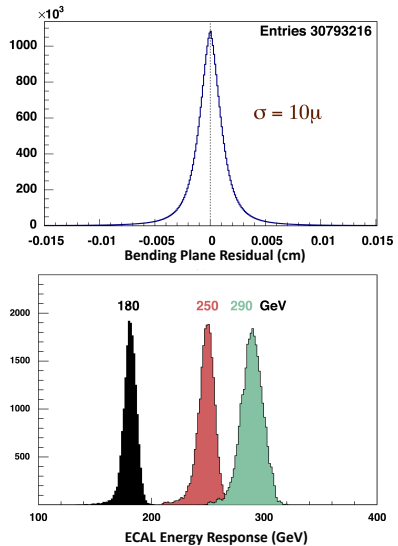

Figure 1: a) Schematic of the AMS-02 detector in its configuration with a superconducting magnet, showing the main detector subsystems (see text). b) Velocity resolution of the Ring Imaging Cherenkov Counter (RICH, above) and rejection power of the Transition Radiation Detector (TRD, below) for protons versus electrons as a function of the electron identification efficiency. c) Distribution of bending plane residuals in all tracker planes (above) and energy response of the electromagnetic calorimeter (ECAL, below) at high energies.

$0.0001 \mathrm{~K} / \mathrm{h}$. With these performance data, the lifetime of the superconducting magnet, limited by the liquid helium reservoir of 2000 liters, was evaluated to $20 \pm 4$ months with the original cryocoolers purchased in 1999, and extrapolated to $28 \pm 6$ months with the latest generation of cryocoolers. The errors of this estimate correspond to systemic uncertainties in the thermal model and uncertainties in heat input from neighboring payloads on the ISS.

\section{Configuration and performance of AMS-02 with the permanent magnet}

In the mean time, the US administration and NASA announced the extension of the ISS lifetime from 2015 to 2010, or eventually even 2028. On the other hand, the Space Shuttle program will definitely be terminated in 2011, eliminating any possibility of returning and refiling the AMS helium supply. While the superconducting magnet was the ideal solution for the originally planned three year stay of AMS on the ISS, the extended lifetime required to search for a more sustainable solution. It was thus decided to fit the permanent magnet used for the AMS-01 pilot mission into a spare AMS-02 vacuum case, to extend the lifetime of the experiment to at least 10 years. This magnet has a smaller central field strength of $0.14 \mathrm{~T}$. The extended lifetime will however provide an improved sensitivity to new high energy phenomena.

In addition to fitting the permanent magnet to the AMS-02 configuration, the lay out of the silicon strip tracker has been changed to recover part of the resolution lost due to the reduced magnetic field, as shown in Fig. 2a). The bottom layer has been moved to the top of the TRD to improve the lever arm on entrance to the magnetic volume. An new layer, composed of spare silicon modules and existing ones recovered from the edge of the spectrometer acceptance, has been added on top of the electromagnetic calorimeter, improving the lever arm upon exit. Replacing the pure sagitta measurement of the superconducting configuration, the new spectrometer lay out uses a 
a)

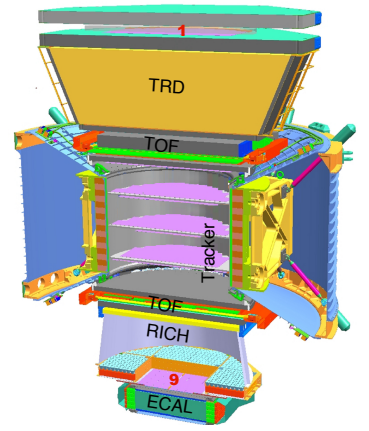

b)

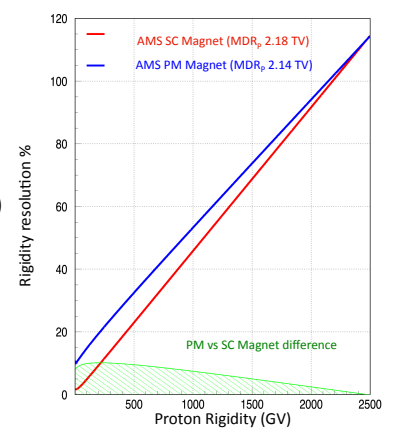

Figure 2: a) Schematic of the AMS-02 detector in its configuration with the permanent magnet, showing the main detector subsystems. Tracker plane 1 has been moved from its position at the spectrometer exit to the top of the TRD. Plane 9 has been added on the bottom before entrance to the ECAL. b) Comparison of the rigidity $r / Z$ resolution of the final flight version of the spectrometer with expectations for the superconducting magnet version.

a)

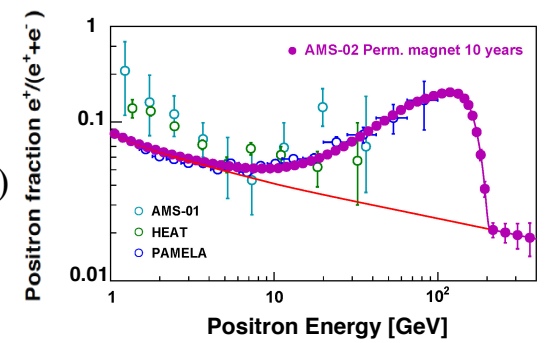

b)

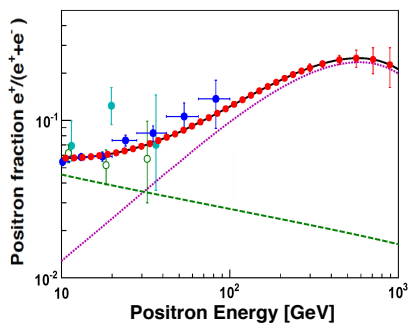

Figure 3: a) Expected positron flux fraction for the AMS-02 final flight version and a 10 years exposure, assuming self-annihilation of $200 \mathrm{GeV}$ neutralinos as dark matter candidates, enhanced to fit previous observations. b) Same for the Nomura-Thaler model N3 with $M_{D M}=3.65 \mathrm{TeV}$ and $E_{F} \simeq 2500$ [5].

combination of sagitta and bend angle determinations to measure momentum. With now 9 sensitive layers, the rigidity resolution using the permanent magnet recovers the one of the superconducting magnet to within $10 \%$ as shown in Fig. 2b). The maximum determined rigidity (MDT) for helium nuclei of this final set up is $3.75 \mathrm{TV}$.

The increased amount of material inside the tracking volume leads to a $10 \%$ rigidity resolution at low momenta, an acceptable compromise given the increased exposure time and the importance of establishing high energy cut offs in particle and antiparticle spectra. The laser alignment control system of AMS-02 only covers the layers inside the magnetic volume. However, accurate alignment of the outermost layers can be achieved in situ using the $10^{4}$ cosmic ray tracks measured per minute in orbit.

The new spectrometer configuration does not compromise the performance of other subdetector components. AMS will thus be able to monitor the composition and spectrum of cosmic rays from $500 \mathrm{MeV}$ to $2 \mathrm{TeV}$ per nucleon, for a period covering a full Solar cycle. Because of its large aperture, it will also be sensitive to transient phenomena. As far as dark matter signals in positron spectra are concerned, Fig. 3 shows two examples of accumulated positron fractions for scenarios 
with supersymmetric dark matter that fit existing observations [5]. The expected sensitivity for residual anti-helium in cosmic rays expected for a long exposure of the final flight configuration remains essentially the same as for the superconducting option. At the end of a 10 year lifetime, the upper limit on a potential strangelet [6] flux, signaled by events with an unusually low $Z / A$ ratio, will reach $3 \times 10^{-12} \mathrm{~cm}^{-2} \mathrm{~s}^{-1} \mathrm{sr}^{-1}$.

\section{Conclusions}

The AMS-02 detector has been successfully integrated with the long lifetime permanent magnet, to match the extended ISS lifecycle. All subsystems are fully functional. The experiment performance at high energies is preserved, especially as far as the rejection power for protons agains positrons and the sensitivity for non standard components of cosmic rays are concerned. The current schedule foreseen operation of the final flight set-up in a CERN test beam from August 7 to 14, 2010. A US Air Force C15 airplane will transport the instrument to Kennedy Space Center, Florida, on August 26, 2010. Space Shuttle mission STS-134, currently scheduled for February 26, 2011, will deliver the detector to the ISS, where it will take data as long as ISS exploitation and lifetime of the embedded electronics allow.

\section{References}

[1] M. Aguilar et al. (AMS collaboration), Phys. Rep. 366 (2002) 331; O. Adriani et al. (PAMELA collaboration), Phys. Rev. Lett. 102 (2009) 051101; O. Adriani et al. (PAMELA collaboration), Phys.Rev.Lett.105 (2010)121101

[2] M. Aguilar et al. (AMS collaboration), Phys. Lett. B484 (2000) 10; M. Aguilar et al. (AMS collaboration), Phys. Lett. B646 (2007) 145; J. Chang et al. (ATTIC collaboration), Nature 456 (2008) 362; O. Adriani et al. (PAMELA collaboration), Nature 458 (2009) 607; S. Coutu et al. (Heat-pbar collaboration), Proc. of the 27th International Cosmic Ray Conference (ICRC 2001), Hamburg, Germany, 7-15 Aug 2001, p. 1687; O. Adriani (PAMELA collaboration), these proceedings.

[3] A.A. Abdo et al. (Fermi LAT collaboration), Phys.Rev.Lett.102 (2009) 181101; A. Aharonian et al. (H.E.S.S. collaboration), Astron.Astrophys.508 (2009) 561

[4] H. Gast et al. (PEBS collaboration), IDM 2008: 7th International Workshop on the Identification of Dark Matter, Stockholm, Sweden, 18-22 Aug 2008, PoS IDM2008 (2008) 023

[5] L. Bergström, J. Edsjö and G. Zaharijas, Phys.Rev.Lett.103 (2009) 031103

[6] E. Witten, Phys. Rev. D30(1984) 272 